\title{
The Dramatic Change in Endoscopic Activities Following the Coronavirus Disease 2019 Outbreak. Is It Evolution?
}

\section{To the Editor:}

The new strain of coronavirus is responsible for the coronavirus disease 2019 (COVID-19) pandemic, starting early 2020. The pandemic has substantially influenced the patterns of endoscopic practice and has necessitated gradual adaptation to the new milieu in the endoscopic field. I read the article with interest regarding the change in endoscopy during COVID-19 outbreak. Lahat et al. ${ }^{1}$ showed that the total endoscopic cases did not differ significantly in January and February between 2020 and the previous two years. However, in March 2020 the numbers were reduced by $44 \%$ compared to previous years. There have been several similar reports from around the world. An Italian study reported reduction of normal endoscopies by $97.6 \%{ }^{2}$ A survey in New York revealed an overall $71 \%$ reduction since the onset of COVID- $19 .^{3}$ The plausible explanation for these phenomena might be the stringent policies initiated, including lockdown, social distancing, and/or self-quarantine. The fear of viral contamination might have resulted in relatively high rates of cancelling or re-scheduling non-urgent endoscopic activities. Lahat et al. ${ }^{1}$ showed a high rate of upper gastrointestinal bleeding during endoscopic procedures conducted during March 2020; however, no further details were provided regarding this unusual observation. Interestingly, Lahat et al. ${ }^{1}$ highlighted that there was no remarkable change in the number of endoscopic retrograde cholangiopancreatographies (ERCPs), although many ERCP

Received: March 5, 2021 Revised: March 17, 2021

Accepted: March 17, 2021

Correspondence: Kook Hyun Kim

Division of Gastroenterology and Hepatology, Department of Internal Medicine, Yeungnam University College of Medicine, 170 Hyeonchung-ro, Nam-gu, Daegu 42415 , Korea

Tel: +82-53-620-3576, Fax: +82-53-654-8386, E-mail: kimkh@yu.ac.kr ORCID: https://orcid.org/0000-0001-7786-7882

(c) This is an Open Access article distributed under the terms of the Creative Commons Attribution Non-Commercial License (http://creativecommons.org/ licenses/by-nc/3.0) which permits unrestricted non-commercial use, distribution, and reproduction in any medium, provided the original work is properly cited. patients usually visit the emergency room due to acute cholangitis, high fever, or abdominal pain. No mention was made of the indications for ERCP in this article.

During the COVID-19 outbreak, telemedicine has become a potentially viable option for ensuring a protective physical barrier between healthcare providers and patients. Virtual technology can deliver medical information including triage, avoid potential viral exposure in the hospital, and minimize evaluation time prior to the endoscopy. ${ }^{4}$

Further evaluation of the change in numbers, indications, and therapeutic modalities of emergent endoscopies can provide a better understanding of changes in endoscopic patterns.

$$
\begin{aligned}
& \text { Conflicts of Interest } \\
& \text { The author has no potential conflicts of interest. } \\
& \text { Funding } \\
& \text { None. } \\
& \text { ORCID https://orcid.org/0000-0001-7786-7882 } \\
& \text { Kook Hyun Kim: Kook Hyun Kim }
\end{aligned}
$$

Division of Gastroenterology and Hepatology, Department of Internal Medicine, Yeungnam University College of Medicine, Daegu, Korea

\section{REFERENCES}

1. Lahat A, Benjamin A. Changes in policy and endoscopic procedures during the 2019 coronavirus disease outbreak: a single center experience. Clin Endosc 2021;54:48-54.

2. Repici A, Pace F, Gabbiadini R, et al. Endoscopy units and the coronavirus disease 2019 outbreak: a multicenter experience from Italy. Gastroenterology 2020;159:363-366.e3.

3. Mahadev S, Aroniadis OS, Barraza L, et al. Impact of the COVID-19 pandemic on endoscopy practice: results of a cross-sectional survey from the New York metropolitan area. Gastrointest Endosc 2020;92:788789.

4. Smith AC, Thomas E, Snoswell CL, et al. Telehealth for global emergencies: Implications for coronavirus disease 2019 (COVID-19). J Telemed Telecare 2020;26:309-313. 


\section{RESPONSE}

We agree with Prof Kook Hyun Kim that patients' fear from exposure to the virus while visiting medical institutions might have added to the reduction in our endoscopic procedures. This was mentioned in the discussion as we related to patients preference to postpone elective procedures.

In our study, we mentioned the total numbers of the specific endoscopic procedure and indications to endoscopies as a percent of the total. Sine total numbers were reduced during the national lockdown, we believed percentages are more appropriate for comparison. As shown in table 1, the percentage of urgent procedures was higher during March 2020, as non-urgent indications (as dyspepsia or heartburn) were postponed.

Along this line, endoscopic retrograde cholangiopancreatography (ERCP) numbers stayed stable since we only perform ERCP for intervention indications. For diagnosis, we perform magnetic resonance cholangiopancreatography.

As mentioned by Prof Kook Hyun Kim, telemedicine is a good option to overcome patients' reluctance and fear from physically arriving at medical centers. We performed telemedicine in our department, studied its' effectiveness, and published our results recently. ${ }^{1}$ Notably, our findings showed telemedicine is more relevant for chronic patients during regular follow-up visits.

Now, after a year of COVID-19 pandemic, we believe that more studies are needed to provide long-term follow-up after its' effect on healthcare policies worldwide.

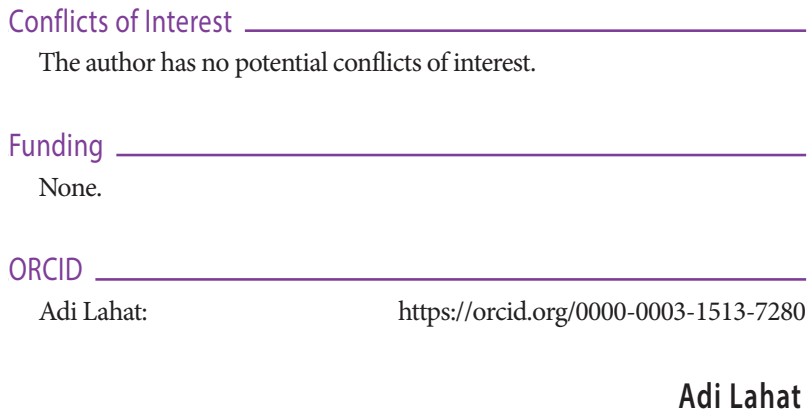

Adi Lahat

Department of Gastroenterology, Sheba Medical Center, Tel Hashomer, Sackler School of Medicine, Tel Aviv University, Tel Aviv, Israel

\section{REFERENCE}

1. Lahat A, Shatz Z. Telemedicine in clinical gastroenterology practice: what do patients prefer? Therap Adv Gastroenterol 2021;14:1756284821989178. 